\title{
EXISTENCE OF UNIVERSAL MEMBERS IN CERTAIN FAMILIES OF BASES OF BANACH SPACES ${ }^{1}$
}

\author{
M. ZIPPIN
}

Abstract. In a recent paper A. Pełczyński proved the existence of universal Schauder bases for several important families of bases. In the present paper some new existence problems are settled. For example, it is proved that the family of boundedly complete bases does not have a universal member.

1. The purpose of this note is to introduce a simple tool which, together with a deep result of A. Pełczyński [3], enables us to settle problems concerning the existence of universal members in several families of bases (see the definitions below). We then use similar methods to verify a conjecture of Pełczyński. We begin with a few definitions. A sequence $\left\{x_{n}\right\}$ of elements of a Banach space $X$ is called a basis if each $x \in X$ has a unique representation $x=\sum_{i=1}^{\infty} t_{i} x_{i}$ where $\left\{t_{i}\right\}$ are scalars. A basis $\left\{x_{n}\right\}$ is called seminormalized [normalized] if $0<\inf _{n}\left\|x_{n}\right\| \leqq \sup _{n}\left\|x_{n}\right\|<\infty\left[\left\|x_{n}\right\|=1\right.$ for all $\left.n\right]$, it is called monotone if for any $n>1$ and scalars $t_{1}, t_{2}, \cdots, t_{n}\left\|\sum_{i=1}^{n-1} t_{i} x_{i}\right\|$ $\leqq\left\|\sum_{i=1}^{n} t_{i} x_{i}\right\|$. Let $\left\{x_{n}\right\}$ and $\left\{y_{n}\right\}$ be bases of the Banach spaces $X$ and $Y$ respectively. Then $\left\{x_{n}\right\}$ is said to be equivalent to $\left\{y_{n}\right\}$ provided that for every sequence $\left\{t_{i}\right\}$ of scalars $\sum_{i=1}^{\infty} t_{i} x_{i}$ converges if and only if $\sum_{i=1}^{\infty} t_{i} y_{i}$ converges. It follows from the closed graph theorem that the bases $\left\{x_{n}\right\}$ and $\left\{y_{n}\right\}$ are equivalent if and only if the map $T: X \rightarrow Y$ defined by $T\left(\sum_{i=1}^{\infty} t_{i} x_{i}\right)=\sum_{i=1}^{\infty} t_{i} y_{i}$ is an isomorphism from $X$ onto $Y$ and therefore two mutually equivalent bases are both seminormalized if one of them is. Assume that $\left\{x_{n}\right\}$ is a basis of $X$ and that $\left\{n_{k}\right\}$ is an increasing sequence of positive integers then the sequence $\left\{x_{n_{k}}\right\}$ is called a subbasis of $\left\{x_{n}\right\}$. It is easy to see that the subbasis $\left\{x_{n_{k}}\right\}$ forms a basis for the subspace it spans in $X$. A subbasis $\left\{x_{n_{k}}\right\}$ is said to be complemented if $\sum_{k=1}^{\infty} t_{n_{k}} x_{n_{k}}$ converges whenever $\sum_{n=i}^{\infty} t_{n} x_{n}$ is convergent. We call a basis $\left\{x_{n}\right\}$ unconditional if for any choice of $\left\{\epsilon_{i}\right\}$ such that $\left|\epsilon_{i}\right|=1, \sum_{i=1}^{\infty} \epsilon_{i} t_{i} x_{i}$ converges whenever $\sum_{i=1}^{\infty} t_{i} x_{i}$ converges. It is clear that every subbasis of an unconditional basis is complemented.

Received by the editors January 26, 1970.

AMS 1969 subject classifications. Primary 4610.

Key words and phrases. Universal basis, seminormalized basis, monotone basis, unconditional basis, complementably universal basis, shrinking basis, boundedly complete basis, equivalent bases, complemented subbasis, norming function, subsequence homogeneous norming function.

1 This research was supported by the NSF Grant GP-12997. 
Let $Q$ be a family of bases (not necessarily in the same space). Following [3] we call a basis $\left\{x_{n}\right\}$ [complementably] universal with respect to $Q$ if any member of $Q$ is equivalent to a [complemented] subbasis of $\left\{x_{n}\right\}$. A. Pełczyński proved the following

Proposition 1 (Theorem 1 of [3]). The following families of bases contain complementably universal elements:

(a) the family of all seminormalized bases;

(b) the family of all seminormalized unconditional bases.

It is the first part of Proposition 1 which we are going to use as our main tool.

2. Let $S$ denote the set of all sequences $\left\{t_{n}\right\}$ of scalars with only finitely many $t_{i}$ different from 0 and let $\phi=\phi\left(t_{1}, t_{2}, \ldots\right)$ be a real function defined on $S$. We call $\phi$ a norming function if the following conditions are satisfied for every scalar $\alpha$ and any two sequences $\left\{t_{n}\right\}$ and $\left\{s_{n}\right\}$ in $S$ :

$\phi\left(t_{1}, t_{2}, \cdots\right) \geqq 0 \quad$ and $\quad \phi(0, \cdots, 0,1,0, \cdots)=1$ for all $n$.

( $n$ zeros)

(2) $\phi\left(t_{1}, t_{2}, \cdots\right)=0$ if and only if $t_{i}=0$ for all $i$.

(3) $\phi\left(\alpha t_{1}, \alpha t_{2}, \cdots\right)=|\alpha| \cdot \phi\left(t_{1}, t_{2}, \cdots\right)$.

(4) $\phi\left(s_{1}+t_{1}, s_{2}+t_{2}, \cdots\right) \leqq \phi\left(s_{1}, s_{2}, \cdots\right)+\phi\left(t_{1}, t_{2}, \cdots\right)$.$$
\phi\left(t_{1}, t_{2}, \cdots, t_{n}, 0,0, \cdots\right) \leqq \phi\left(t_{1}, t_{2}, \cdots, t_{n}, t_{n+1}, 0,0, \cdots\right)
$$

for all $n$.

LeMma 1. Let $\left\{x_{n}\right\}$ be a normalized basis of a Banach space $X$, let $\phi=\phi\left(t_{1} t_{2} \cdots\right)$ be a norming function and put

$$
\Phi\left(\left\{s_{n}\right\}\right)=\sup _{n} \phi\left(s_{1}, s_{2}, \cdots, s_{n}, 0,0, \cdots\right)
$$

for any sequence $\left\{s_{n}\right\}$ of scalars. Let $X_{\phi}=\left\{\sum_{i=1}^{\infty} t_{i} x_{i} \in X \mid \Phi\left(\left\{t_{n}\right\}\right)\right.$ $<\infty\}$.

Then $X_{\phi}$ is a Banach space under the norm defined for $x=\sum_{i=1}^{\infty} t_{i} x_{i}$ by $\mid\|x\|=\max \left\{\|x\|, \Phi\left(\left\{t_{n}\right\}\right)\right\}$.

Proof. It is obvious that $X_{\phi}$ is a normed space. To prove completeness, let $y_{k}=\sum_{i=1}^{\infty} \alpha_{i}^{k} x_{i}$ be a Cauchy sequence in $X_{\phi}$. Then, clearly, $\left\{y_{k}\right\}$ is a Cauchy sequence in $X$ and hence has a limit $y=\sum_{i=1}^{\infty} \alpha_{i} x_{i}$ in $X$. As is well known (see e.g. [2, p. 69 (4)]) there is an $M \geqq 1$ such that $\left\|\sum_{i=1}^{n} \alpha_{i} x_{i}\right\| \leqq M\left\|\sum_{i=1 !}^{n+k} \alpha_{i} x_{i}\right\|$ for all natural $n$ and $k$ and every sequence $\alpha_{1}, \alpha_{2}, \cdots, \alpha_{n+k}$ of scalars. Given $\epsilon>0$ choose $k=k(\epsilon)$ such 
that ||$\left|y_{p}-y_{q}\right| \mid<M^{-1} \cdot \epsilon$ whenever $p, q>k$. It follows that for each $n$

$$
\left\|\sum_{i=1}^{n}\left(\alpha_{i}^{p}-\alpha_{i}^{q}\right) x_{i}\right\| \leqq M \cdot \max \left\{\left\|\sum_{i=1}^{\infty}\left(\alpha_{i}^{p}-\alpha_{i}^{q}\right) x_{i}\right\|, \Phi\left(\left\{\alpha_{i}^{p}-\alpha_{i}^{q}\right\}\right)\right\} \leqq \epsilon .
$$

Letting $q$ tend to $\infty$ we get

$$
\left\|\sum_{i=1}^{n}\left(\alpha_{i}^{p}-\alpha_{i}\right) x_{i}\right\| \mid \leqq \text { if } p>k .
$$

Since ||$\left|\sum_{i=1}^{\infty}\left(\alpha_{i}^{p}-\alpha_{i}\right) x_{i}\right|\left\|\leqq \sup _{n}\left|\left\|\sum_{i=1}^{n}\left(\alpha_{i}^{p}-\alpha_{i}\right) x_{i} \mid\right\|\right.\right.$ it follows from (6) that ||$\left|\sum_{i=1}^{\infty}\left(\alpha^{p}-\alpha_{i}\right) x_{i}\right| \| \leqq \epsilon$, which implies that $y=\sum_{i=1}^{\infty} \alpha_{i} x_{i}$ $\in X_{\phi}$ and that $\left\|y_{k}-y\right\| \| \underset{k \rightarrow \infty}{\longrightarrow} 0$. This completes the proof.

Given a normalized basis $\left\{x_{n}\right\}$ in a Banach space $X$, of particular interest is the norming function $\phi$ defined for each $\left\{t_{n}\right\} \in S$ by

$$
\phi\left(t_{1}, t_{2}, \cdots, t_{n}, \cdots\right)=\sup _{\mu \in \Sigma}\left\|\sum_{i \in \mu} t_{i} x_{i}\right\|
$$

where $\Sigma$ is the collection of all finite subsets of positive integers. By Lemma $1, X_{\phi}$ is a Banach space. Let us denote by $\bar{x}_{n}$ the element $x_{n}$ when regarded as a member of $X_{\phi}$, then $\left\{\bar{x}_{n}\right\}$ is an unconditional basis of $X_{\phi}$. Indeed, as is well known (see e.g. $[2$, p. 59]) a series $\sum_{i=1}^{\infty} t_{i} \bar{x}_{i}$ converges unconditionally if and only if

$$
\sup _{\mu \in \Sigma}\left\|\left|\sum_{i \in \mu} t_{i} \bar{x}_{i} \|\right|<\infty\right.
$$

but since

$$
\sup _{\mu \in \Sigma}\left\||| \sum_{i \in \mu} t_{i} \bar{x}_{i}\right\|=\sup _{\mu \in \Sigma}\left\|\sum_{i \in \mu} t_{i} \bar{x}_{i}\right\|=\Phi\left(\left\{t_{n}\right\}\right)
$$

and this is a finite number whenever $\sum_{i=1} l_{i} x_{i} \in X_{\phi}$ we finally get that $\left\{\bar{x}_{n}\right\}$ is an unconditional basis. Note that $X_{\phi}$ consists of all elements $x=\sum_{i=1}^{\infty} t_{i} x_{i}$ of $X$ for which the series converges unconditionally and therefore some properties of the space $X_{\phi}$ may show the "extent of unconditionality" of the basis $\left\{x_{n}\right\}$ of $X$.

The above observations lead us to the following

Alternative Proof of Proposition 1(b). Let $\left\{x_{n}\right\}$ be a normalized universal basis for the family of all seminormalized bases. (The existence of such a basis may be assumed by Proposition 1(a).) Let $\phi$ be defined by (7) and consider the basis $\left\{\bar{x}_{n}\right\}$ of $X_{\phi}$ as above. We have shown that $\left\{\bar{x}_{n}\right\}$ is unconditional and we now claim that it forms a universal basis for all seminormalized unconditional bases. 
Indeed, let $\left\{y_{k}\right\}$ be a seminormalized unconditional basis. Then, by universality of $\left\{x_{n}\right\}$, there is a subbasis $\left\{x_{n_{k}}\right\}$ equivalent to $\left\{y_{k}\right\}$. Since $\||x|\| \geqq\|x\|$ for all $x \in X_{\phi}$, the convergence of $\sum_{k=1}^{\infty} t_{k} \bar{x}_{n_{k}}$ in $X_{\phi}$ implies the convergence of $\sum_{k=1}^{\infty} t_{k} x_{n_{k}}$ in $X$ and hence, by equivalence, also $\sum_{k=1}^{\infty} t_{k} y_{k}$ converges. Conversely, if $\sum_{k=1}^{\infty} t_{k} y_{k}$ converges it converges unconditionally and, by equivalence, $\sum_{k=1}^{\infty} t_{k} x_{n_{k}}$ converges unconditionally in $X$. It follows that $\sum_{k=1}^{\infty} t_{k} x_{n_{k}} \in X_{\phi}$ which implies the convergence of $\sum_{k=1}^{\infty} t_{k} \bar{x}_{n_{k}}$ in $X_{\phi}$. We have shown that there is a subbasis $\left\{\bar{x}_{n_{k}}\right\}$ of $\left\{\bar{x}_{n}\right\}$ which is equivalent to $\left\{y_{k}\right\}$ and thus the universality of $\left\{\bar{x}_{n}\right\}$ is proved.

A norming function $\phi$ is called subsequence homogeneous if, for any $\left\{t_{n}\right\} \in S, \phi\left(t_{1}, t_{2}, \cdots\right)=\phi\left(s_{1}, s_{2}, \cdots\right)$ for every sequence $\left\{s_{n}\right\} \in S$ having the same number, $k$ say, of nonzero terms such that for each $1 \leqq i \leqq k$ the $i$ th nonzero term of $\left\{s_{n}\right\}$ is equal to the $i$ th nonzero term of $\left\{t_{n}\right\}$. The following result independently observed by Pełczyński shows the existence of universal members in several families of bases:

Proposition 2. Let $\phi$ be a subsequence homogeneous norming function and let $a_{\phi}$ denote the family of all seminormalized bases $\left\{y_{k}\right\}$ such that the convergence of $\sum_{k=1}^{\infty} t_{k} y_{k}$ implies that

$$
\Phi\left(\left\{t_{n}\right\}\right)=\sup _{n} \phi\left(t_{1}, t_{2}, \cdots, t_{n}, 0,0, \cdots\right)<\infty .
$$

Then $a_{\phi}$ contains a normalized universal member.

Proof. Let $\left\{x_{n}\right\}$ be a normalized basis (in some space $X$ ) which is universal with respect to the family of all seminormalized bases. Construct the space $X_{\phi}$ as in Lemma 1 and denote by $\bar{x}_{n}$ the element $x_{n}$ when regarded as a member of $X_{\phi}$, then, as is easy to see, $\left\|\left|\bar{x}_{n}\right|\right\|=1$ and $\left\{\bar{x}_{n}\right\} \in a_{\phi}$. Let $\left\{z_{k}\right\} \in a_{\phi}$ and let $\left\{x_{n_{k}}\right\}$ be a subbasis of $\left\{x_{n}\right\}$ (in $X$ ) equivalent to $\left\{z_{k}\right\}$. If $\sum_{k=1}^{\infty} t_{k} z_{k}$ converges then $\Phi\left(\left\{t_{k}\right\}\right)<\infty$ and, because $\phi$ is subsequence homogeneous, $\sum_{k=1}^{\infty} t_{k} x_{n_{k}} \in X_{\phi}$ and therefore $\sum_{k=1}^{\infty} t_{k} \bar{x}_{n_{k}}$ converges in $X_{\phi}$. Conversely, if $\sum_{k=1}^{\infty} t_{k} \bar{x}_{n_{k}}$ converges in $X_{\phi}$ then $\sum_{k=1}^{\infty} t_{k} x_{n_{k}}$ converges in $X$ (note that $\|x\|\|\leqq x\|$ for all $x \in X_{\phi}$ ) and, by equivalence, $\sum_{k=1}^{\infty} t_{k} z_{k}$ is convergent. It follows that $\left\{z_{k}\right\}$ is equivalent to $\left\{\bar{x}_{n_{k}}\right\}$ in $X_{\phi}$, and this proves the universality of the basis $\left\{x_{n}\right\}$ of $X_{\phi}$.

REMARK 1. In a private communication A. Pełczyński remarked that by using Proposition 1(a), Proposition 2 and duality one can prove the existence of a universal member in the family $a_{\phi}^{*}$ of all bases $\left\{y_{n}\right\}$ such that $\sum \alpha_{i} y_{i}$ converges whenever $\Phi\left(\left\{\alpha_{n}\right\}\right)<\infty$.

3. A basis $\left\{x_{n}\right\}$ of a Banach space $X$ is called shrinking if the biorthogonal functionals $\left\{x_{n}^{*}\right\}$ (defined by $x_{n}^{*}\left(x_{k}\right)=\delta_{n, k}$ ) span the 
dual space $X^{*}$. It is called boundedly complete if $\sum_{k=1}^{\infty} t_{k} x_{k}$ converges whenever $\sup _{n}\left\|\sum_{k=1}^{n} t_{k} x_{k}\right\|<\infty$. A. Pełczyński proves [3, Theorem 4] that there is no shrinking basis universal for the family of all shrinking bases. It is conjectured in [3, p. 267] that a similar result holds for boundedly complete bases. Before verifying this conjecture we need the following

Lemma 2. Let $\left\{x_{n}\right\}$ be a basis of a Banach space $X$ and define $\phi$ by (7). Construct the space $X_{\phi}$ as before and denote by $\bar{x}_{k}$ the element $x_{k}$ when regarded as an element of $X_{\phi}$. Then $\left\{\bar{x}_{n}\right\}$ is a boundedly complete basis of $X_{\phi}$ if any one of the following conditions is satisfied:

(a) $\left\{x_{n}\right\}$ is a boundedly complete basis of $X$.

(b) $X$ is isomorphic to a dual space $Y^{*}$.

(c) $X$ is weakly sequentially complete.

(d) $X$ does not have a subspace isomorphic to $c_{0}$ (=the space of all sequences $\lambda=\left\{\lambda_{n}\right\}$ of scalars converging to 0 with $\left.\|\lambda\|=\max _{n}\left|\lambda_{n}\right|\right)$.

Proof. We first note that any one of the first three conditions implies (d). Indeed (a) $\Rightarrow$ (b) by [2, p. 67, Theorem 1, and p. 70, Lemma 2]. (b) $\Rightarrow(d)$ because by $\left[1\right.$, Theorem 4] $c_{0}$ cannot be isomorphic to a subspace of a separable dual space. $(\mathrm{c}) \Rightarrow(\mathrm{d})$ because $c_{0}$ is not weakly sequentially complete. Now assume that (d) holds. If $\left\{\bar{x}_{n}\right\}$ is not a boundedly complete basis of $X_{\phi}$ then by the proof of Theorem 2 of p. 74 of [2] there is a sequence of blocks

$$
z_{k}=\sum_{i=p_{k+1}}^{p_{k+1}} \beta_{i} \bar{x}_{i}, \quad k=1,2, \cdots,
$$

such that $\left\{p_{k}\right\}$ is an increasing sequence of positive integers $\inf _{k}\left\|z_{k}\right\|=\delta>0$ and $\left\{z_{k}\right\}$ is a basis (for its span) equivalent to the unit vectors of $c_{0}$. It follows from the definition of the norm \|\|$\cdot \|$ that for each $k$ there is a subset $\mu_{k} \in\left\{p_{k}+1, p_{k}+2, \cdots, p_{k+1}\right\}$ such that $\left\|\sum_{i \in \mu_{k}} \beta_{i} x_{i}\right\| \geqq \delta / 2$. We claim that the elements $u_{k}=\sum_{i \in \mu_{k}} \beta_{i} x_{i}$, $k=1,2, \cdots$, form a basis (for the subspace they span in $X$ ) which is equivalent to the unit vector basis of $c_{0}$. Proving this claim which obviously contradicts (d) we will establish the assertion of Lemma 2.

It is well known and easy to show that any sequence of blocks (like $u_{k}$ ) with respect to a given basis of a Banach space $X$ forms a basis for the subspace it spans in $X$. It is also clear that in view of the inequality $\inf _{k}\left\|u_{k}\right\| \geqq \delta / 2$ the convergence of $\sum_{i=1}^{\infty} \alpha_{i} u_{i}$ in $X$ implies that $\alpha_{i \rightarrow i \rightarrow \infty} 0$. To prove the converse, let $\alpha_{i \rightarrow \infty} \vec{\longrightarrow} 0$. Then $\sum_{i=1}^{\infty} \alpha_{i} z_{i}$ converges in $X_{\phi}$ (because of the equivalence of $\left\{z_{k}\right\}$ to the unit vector basis of $c_{0}$ ). It follows from the definition of the norm 
$\left|\|\cdot \mid\|\right.$ that for any $\left.x \in X_{\phi}\right|\|x \mid\| \geqq\|x\|$ and also that \|\|$\sum_{i \in \nu} \gamma_{i} \bar{x}_{i} \|$ $\geqq\left\||| \sum_{i \in \mu} \gamma_{i} \bar{x}_{i} \mid\right\|$ for any $\mu \subset \nu \in \Sigma$ and every $\sum_{i \in \nu} \gamma_{i} \bar{x}_{i} \in X_{\phi}$. Therefore for any $m<n$,

$$
\begin{aligned}
\left\|\sum_{i=m}^{n} \alpha_{i} z_{i}\right\| & =\left\|\mid \sum_{i=m}^{n} \alpha_{i}\left(\sum_{j=p_{i}+1}^{p_{i+1}} \beta_{j} \bar{x}_{j}\right)\right\| \\
& \geqq\left\|\mid \sum_{i=m}^{n} \alpha_{i}\left(\sum_{j \in \mu_{i}} \beta_{j} \bar{x}_{j}\right)\right\|\|\geqq\| \sum_{i=m}^{n} \alpha_{i} u_{i} \|
\end{aligned}
$$

and hence $\sum_{i=1}^{\infty} \alpha_{i} u_{i}$ converges in $X$. This concludes the proof of Lemma 2.

Let $X$ and $Y$ be Banach spaces and $1 \leqq p \leqq \infty$. Then $X \oplus_{p} Y$ denotes the space of all pairs $(x, y)$ where $x \in X, y \in Y$, with norm

$$
\begin{aligned}
\|(x, y)\| & =\left(\|x\|^{p}+\|y\| p\right)^{1 / p} & & \text { if } 1 \leqq p<\infty, \\
& =\max \{\|x\|,\|y\|\} & & \text { if } p=\infty .
\end{aligned}
$$

Following [3], given a countable family $\left\{X_{\alpha}\right\}_{\alpha \in A}$ of Banach spaces we denote by $\left(P_{\alpha \in A} X_{\alpha}\right)_{2}$ the space of all functions $x=x(\alpha)$ on $A$ with $x(\alpha) \in X_{\alpha}$ for every $\alpha \in A$ such that $\|x\|=\left(\sum_{\alpha \in A}\|x(\alpha)\|^{2}\right)^{1 / 2}$ $<\infty$. Let $\omega_{1}$ denote the first uncountable ordinal and, as in [3], construct by transfinite induction a family of spaces $X_{\alpha}$ for all ordinals $\alpha<\omega_{1}$ as follows: $X_{0}=l_{2}, X_{\alpha}=X_{\beta} \oplus_{2} l_{1}$ if $\alpha=\beta+1$ and $X_{\alpha}=\left(P_{\beta<\alpha} X_{\beta}\right)_{2}$ if $\alpha$ is a limit. It is easy to prove that, for each $\alpha<\omega_{1}, X_{\alpha}$ is a reflexive Banach space having a normalized unconditional basis $\left\{u_{\alpha, k}\right\}_{k=1}^{\infty}$ which is both shrinking and boundedly complete (see e.g. [ 2 p. 71]). Moreover, for each $\alpha<\omega_{1}$ the biorthogonal functionals $\left\{u_{\alpha, k}^{*}\right\}_{k=1}^{\infty}$ of $\left\{u_{\alpha, k}\right\}_{k=1}^{\infty}$ form a basis for $X_{\alpha}^{*}$ sharing the same properties. In [3] Pełczyński is using the following result of Szlenk [4].

Let $F$ be a Banach space which contains for each $\alpha<\omega_{1}$ a subspace isomorphic to $X_{\alpha}$. Then $F$ is not separable.

This result enables us to prove Pełczyński's conjecture namely

THEOREM. The following families of bases do not contain a universal member:

(a) The family of all seminormalized boundedly complete bases;

(b) The family of all seminormalized bases of spaces isomorphic to dual spaces;

(c) The family of all seminormalized bases of weakly sequentially complete spaces;

(d) The family of all seminormalized bases of spaces which do not contain a subspace isomorphic to $c_{0}$. 
Proof. Suppose that a basis $\left\{z_{n}\right\}$ (not necessarily seminormalized) in some Banach space $X$ is universal for one of the above mentioned families and belongs to this family. Put $x_{n}=z_{n} /\left\|z_{n}\right\|$, then the basis $\left\{x_{n}\right\}$ need not be universal with respect to the whole family, $Q$ say, under consideration however, it is universal for all unconditional members of $a$. (This follows easily from the fact that if $\left\{y_{n}\right\}$ is an unconditional basis and $\left\{\alpha_{n}\right\}$ is a sequence of scalars with $0<\inf \left|\alpha_{n}\right|$ $\leqq \sup \left|\alpha_{n}\right|<\infty$ then $\left\{\alpha_{n} y_{n}\right\}$ is a basis equivalent to $\left\{y_{n}\right\}$.) Define $\phi$ by (7) and construct the space $X_{\phi}$ and the unconditional basis $\left\{\bar{x}_{n}\right\}$ as in $\$ 2$. We know that a series $\sum_{i=1}^{\infty} t_{i} \bar{x}_{i}$ converges in $X_{\phi}$ if and only if it converges unconditionally in $X$ and hence for each unconditional member $\left\{y_{n}\right\}$ of $a$ there is a subbasis of $\left\{\bar{x}_{n}\right\}$ equivalent to $\left\{y_{n}\right\}$. In particular, for each $\alpha<\omega_{1}$ the basis $\left\{u_{\alpha, k}^{*}\right\}_{k=1}^{\infty}$ of $X_{\alpha}^{*}$ being a member of $Q$ is equivalent to some subbasis $\left\{\bar{x}_{n(\alpha, k)}\right\}_{k=1}^{\infty}$ of $\left\{\bar{x}_{n}\right\}$. It follows from (7) and Lemma 2 that $\left\{\bar{x}_{n}\right\}$ is a boundedly complete monotone basis and therefore, by [2, p. 70, Lemma 2], the biorthogonal functionals $\left\{\bar{x}_{n}^{*}\right\}$ of $\left\{\bar{x}_{n}\right\}$ form an unconditional shrinking basis of the subspace $F$ they span in $X_{\phi}^{*}$ and $F^{*}$ is isometric to $X_{\phi}$. Since every subbasis of an unconditional basis is complemented it is easy to see that for each $\alpha<\omega_{1}$ the subbasis $\left\{\bar{x}_{n(\alpha, k)}^{*}\right\}_{k=1}^{\infty}$ of $\left\{\bar{x}_{n}^{*}\right\}$ is equivalent to the basis $\left\{u_{\alpha, k}\right\}_{k=1}^{\infty}$ of $X_{\alpha}$ and hence $F$ contains a subspace isomorphic to $X_{\alpha}$. In view of Szlenk's result this contradicts the separability of $X_{\phi}=F^{*}$ and the Theorem is thus proved.

REMARK 2. Using entirely different methods P. Wojtaszczyk [5] has recently proved the Theorem for the family (a).

\section{REFERENCES}

1. C. Bessaga and A. Pełczyński, On bases and unconditional convergence of series in Banach spaces, Studia Math. 17 (1958), 151-164. MR 22 \#5872.

2. M. M. Day, Normed linear spaces, Ergebnisse der Mathematik und ihrer Grenzgebiete, Heft 21, Springer-Verlag, Berlin, 1958. MR 20 \#1187.

3. A. Pełczyński, Universal bases, Studia Math. 32 (1969), 247-268.

4. W. Szlenk, The non-existence of a separable reflexive Banach space universal for all separable reflexive Banach spaces, Studia Math. 30 (1968), 53-61. MR 37 \#3327.

5. P. Wojtaszczyk, On separable Banach spaces containing all separable reflexive Banach spaces, Studia Math. (to appear).

University of California, Berkeley, California 94720 